University of Nebraska - Lincoln

DigitalCommons@University of Nebraska - Lincoln

\title{
Impact of solar Ultraviolet-B on the proteome in soybean lines differing in flavonoid contents
}

\author{
Chenping $\mathrm{Xu}$ \\ University of Maryland - College Park \\ Joe H. Sullivan \\ University of Maryland - College Park \\ Wesley M. Garrett \\ USDA-ARS, wesley.garrett@ars.usda.gov \\ Thomas J. Caperna \\ United States Department of Agriculture- Agricultural Research Service \\ Savithiry Natarajan \\ United States Department of Agriculture-Agricultural Research Service, natarajs@ba.ars.usda.gov
}

Follow this and additional works at: https://digitalcommons.unl.edu/usdaarsfacpub

Part of the Agricultural Science Commons

\footnotetext{
Xu, Chenping; Sullivan, Joe H.; Garrett, Wesley M.; Caperna, Thomas J.; and Natarajan, Savithiry, "Impact of solar Ultraviolet-B on the proteome in soybean lines differing in flavonoid contents" (2008).

Publications from USDA-ARS / UNL Faculty. 696.

https://digitalcommons.unl.edu/usdaarsfacpub/696
}

This Article is brought to you for free and open access by the U.S. Department of Agriculture: Agricultural Research Service, Lincoln, Nebraska at DigitalCommons@University of Nebraska - Lincoln. It has been accepted for inclusion in Publications from USDA-ARS / UNL Faculty by an authorized administrator of DigitalCommons@University of Nebraska - Lincoln. 


\title{
Impact of solar Ultraviolet-B on the proteome in soybean lines differing in flavonoid contents
}

\author{
Chenping Xu ${ }^{\text {a }}$, Joe H. Sullivan ${ }^{\text {a, }}$, Wesley M. Garrett ${ }^{\mathrm{b}}$, \\ Thomas J. Caperna ${ }^{c}$, Savithiry Natarajan ${ }^{\text {d,* }}$ \\ ${ }^{a}$ University of Maryland, Department of Plant Science and Landscape Architecture, College Park, MD 20742, United States \\ ${ }^{\mathrm{b}}$ US Department of Agriculture, Agricultural Research Service, Biotechnology and Germplasm Laboratory, Beltsville, MD 20705, United States \\ ${ }^{\mathrm{c}}$ US Department of Agriculture, Agricultural Research Service, Growth Biology Laboratory, Beltsville, MD 20705, United States \\ ${ }^{\mathrm{d}}$ US Department of Agriculture, Agricultural Research Service, Soybean Genomics and Improvement Laboratory, PSI, Beltsville, MD 20705 , United States
}

Received 16 January 2007; received in revised form 4 May 2007

Available online 23 July 2007

\begin{abstract}
Two-dimensional polyacrylamide gel electrophoresis (2-D PAGE) was used to systematically investigate the impact of solar ultraviolet-B (UV-B) radiation on the soybean leaf proteome. In order to investigate the protective role of flavonoids against UV-B, two isolines of the Clark cultivar (the standard line with moderate levels of flavonoids and the magenta line with reduced flavonoids) were grown in the field with or without natural levels of UV-B. The 12-day-old first trifoliates were harvested for proteomic analysis. More than 300 protein spots were reproducibly resolved and detected on each gel. Statistical analysis showed that 67 protein spots were significantly $(P<0.05)$ affected by solar UV-B. Many more spots were altered by UV-B in the magenta line than in the standard line. Another 12 protein spots were not altered by UV-B but showed significantly $(P<0.05)$ different accumulations between the two lines, and for most spots the line-specific differences were also observed under UV-B exclusion. Most of the differentially accumulated spots were identified by mass spectrometry. The proteins were quite diverse, and were involved in metabolism, energy, protein destination/storage, protein synthesis, disease/defense, transcription, and secondary metabolism. The results suggest that high levels of flavonoids lead to a reduction in UV-B sensitivity at the proteomic level.
\end{abstract}

Published by Elsevier Ltd.

Keywords: 2-D PAGE; Flavonoids; Mass spectrometry; Soybean; Proteomics; UV-B

\section{Introduction}

Increases in chlorofluorocarbons in the atmosphere may deplete the earth's stratospheric ozone layer (Molina and Rowland, 1974), and a decrease in the ozone column lead to an increase in the levels of ultraviolet-B (UV-B: 280-320 nm) radiation that reach the earth's surface (Blumthaler and Amback, 1990; Gleason et al., 1993). Although UV-B radiation has important regulatory and photomorphogenic roles (Ballare et al., 1995), excessive

\footnotetext{
${ }^{*}$ Corresponding author. Fax: +1 3015045728.

E-mail address: natarajs@ba.ars.usda.gov (S. Natarajan).
}

UV-B radiation is clearly harmful. In general, a high level of UV-B causes reduced photosynthesis and growth (Ruhland et al., 2005; Germ et al., 2005), oxidative damages (Yannarelli et al., 2006), and damage to DNA (Bray and West, 2005).

Plants possess an array of adaptive responses to UV-B that allow them to prevent, mitigate or repair UV-B damage. We still do not have a complete understanding of the molecular bases of these responses, but they generally are the result of signal perception by receptor molecules and transduction of a response signal to the cellular machinery, a part of which may regulate gene expression. Many researchers have studied the effects of UV-B on gene expression and have identified a number of UV-B 
responsive genes in plants. For example, photosynthetic genes may be down-regulated (A-H-Mackerness et al., 2001; Jordan et al., 1998; Surplus et al., 1998), while pathogenesis-related genes, the defencin gene (PDF 1,2) (A-H-Mackerness et al., 1999, 2001), genes for flavonoids biosynthesis (A-H-Mackerness et al., 2001; Chappell and Hahlbrock, 1984) and antioxidant enzymes (A-H-Mackerness et al., 1998; Willekens et al., 1994) may be up-regulated by UV-B. Using microarray analysis, Casati and Walbot (2004) and Ulm et al. (2004) identified more than 100 UV-B responsive genes in maize and Arabidopsis, respectively. However, most studies were conducted indoors under artificial condition with unrealistically high UV-B radiation as well as low ultraviolet-A (UV-A: 320 $400 \mathrm{~nm}$ ) and photosynthetically active radiation (PAR: $400-700 \mathrm{~nm}$ ). Responses of plants in controlled conditions may differ from those in field conditions, because of the different levels of UV-A and PAR (Krizek, 2004). Casati and Walbot (2003) examined the response of gene expression in maize to solar UV-B under field conditions, and found several photosynthesis-associated genes were decreased and antioxidant-associated genes were increased. Also, the genes involved in the fatty acid metabolism and oxylipin biosynthesis were increased by solar UV-B (Izaguirre et al., 2003). These results are the most comprehensive data currently available on the effects of solar UV-B on plant gene expression.

However, these studies only looked at mRNA level, which may not necessarily translate into the quantity and quality of the final gene products, i.e. the proteins. There is a loose correlation between mRNA and protein levels, especially for chloroplast genes, which are usually controlled at the post-transcriptional level (Jordan et al., 1992; A-H-Mackerness et al., 1997). Moreover, many proteins undergo post-translational modifications (PTM) such as removal of signal peptides, phosphorylation and glycosylation, which are extremely important for protein activities and subcellular localizations. Therefore, changes at the mRNA level alone may not adequately assess the response to UV-B, and it is necessary to study the effects of UV-B at the protein level. There has been only limited research on the effects of UV-B on proteins, and most of this research focused on a single protein, such as PR-1 (Green and Fluhr, 1995), glutathione reductase, ascorbate peroxidase, superoxide dismutase (Rao et al., 1996) or nitrite reductase (Migge et al., 1998).

Two-dimensional polyacrylamide gel electrophoresis (2D PAGE) is a sensitive and powerful technique for resolving hundreds of proteins in parallel. Combined with mass spectrometry (MS), it allows rapid and reliable protein identification and can provide information about abundance and PTM. In recent years, proteomic-based technologies have been successfully applied to the systematic study of the proteomic responses in many plant species to a wide range of abiotic stresses, including drought (Pinheiro et al., 2005), nutrition deficiency (Alves et al., 2006), temperature (Yan et al., 2006; Sule et al., 2004), oxidative stress (Wang et al., 2004), herbicide (Castro et al., 2005), wound (Shen et al., 2003), anoxia (Chang et al., 2000), salt (Yan et al., 2005) and heavy metal (Labra et al., 2006). Casati et al. (2005) have used proteomic technologies to investigate the effects of UV-B on the proteome of the maize leaf. They found that UV-B radiation regulated the accumulation of 178 protein spots and phosphorylated pyruvate phosphate dikinase.

Soybean (Glycine max) provides an inexpensive source of protein for human consumption and the animal industry and it has been the dominant oilseed produced since the 1960. Soybean genotypes exhibit a wide range in sensitivity to UV-B radiation due in part to differences in flavonoid content (Middleton and Teramura, 1993; Reed et al., 1992), since the flavonoids in the leaf epidermis can screen out UV-B radiation (Schmelzer et al., 1988; Robberecht and Caldwell, 1983). The purpose of this UV-B exclusion study was to systematically examine the effects of solar UV-B on the soybean leaf proteome and to investigate whether flavonoids afforded protection against solar UV$\mathrm{B}$ under field conditions. Two soybean isolines with different flavonoid content were used: the Clark standard line that produces moderate levels of flavonol glycoside, and the Clark magenta line that has reduced flavonol glycoside levels (Buzzell et al., 1977).

\section{Results}

\subsection{2-D PAGE and quantitative analysis}

More than 300 protein spots were clearly separated and detected by 2-D PAGE despite the predominance of ribulose bisphosphate carboxylase/oxygenase (Rubisco). The 2-D PAGE gels were reproducible and had well-separated spots, although as is frequently observed with proteome analysis, the dynamic range of protein accumulation was very large (Wilson et al., 2002; Watson et al., 2003). A representative gel image is presented in Fig. 1a. Only spots that were significantly affected by UV-B, or spots that had different intensity between the two lines were further analyzed. A total of 79 spots were selected, and no interaction between UV-B and line was detected for these 79 spots. The statistical data for the 79 spots are listed in Table 1, and magnified regions of several differentially accumulated proteins are presented in Fig. 1b. The 79 selected spots were divided into three groups. Spots in Group I are those that were increased by solar UV-B; this group had 31 spots (spots 1-31; Fig. 1; Table 1). Group II includes 36 spots that were decreased by solar UV-B (spots 32-67; Fig. 1; Table 1). Group III includes 12 spots that had different intensity between the two lines but were not altered by UV-B (spots 68-79; Fig. 1; Table 1). The accumulations of a total of 67 spots were altered by solar UV-B, and the total number of spots that increased in abundance was similar to the number of spots that decreased. 


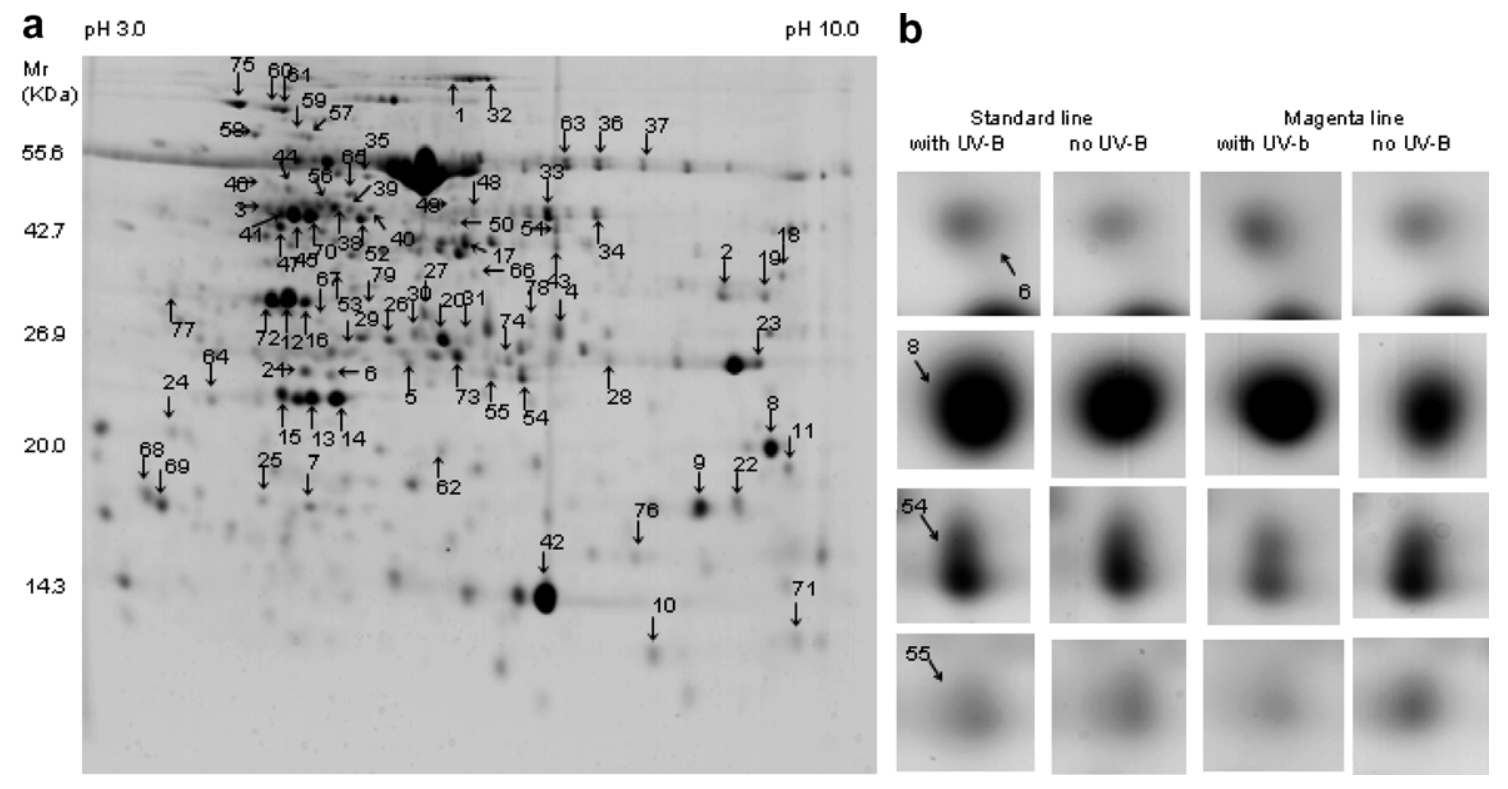

Fig. 1. 2-D PAGE gel image and magnified regions of some protein spots. Arrows indicate protein spots that were differentially accumulated between different UV-B treatments or between different lines $(P<0.05)$. Numbers correlate with protein identifications and statistical analysis results listed in Tables 1-3. (a) Representative 2-D PAGE gel image of soybean leaves (cv. Clark, standard line) grown under Teflon shelter. (b) Magnified regions of several differentially accumulated proteins in two lines of soybean growing under different UV-B conditions.

In group I, only four spots (spots 5, 27, 29, 30) were increased in both lines, 23 spots (spots $2,4,6-9,11-16$, $18-26,28,31)$ were increased only in the magenta line, and four spots (spots $1,3,10,17$ ) increased only in the standard line (Table 1). In group II, 14 spots (spots 32, $38-40,44,45,47,49,51,56-59,61)$ were decreased in both lines, and 16 spots (spots $35-37,41-43,46,48,50,52,54$, $55,60,64-66)$ were decreased only in the magenta line, whereas 6 spots (spots 33, 34, 53, 62, 63, 67) decreased only in the standard line (Table 1). Many more proteins were responsive to solar UV-B in the magenta line than in the standard line. In group III, 11 spots (spots 68-70, 72-79) showed different intensities between the two lines even with no UV-B treatment (Table 1).

\subsection{Identification of differentially accumulated proteins}

Of the 79 spots, 56 had been identified previously ( $\mathrm{Xu}$ et al., 2006). The remaining 23 spots were digested with trypsin and subjected to matrix-assisted laser desorption/ ionization-time of flight (MALDI-TOF) MS. Only one spot (spot 3, Rubisco activase) could be identified in this way. The other 22 spots were further analyzed by liquid chromatography tandem mass spectrometry (LC-MS/ MS), and 16 of these spots could be identified (Table 2). Both the theoretical and experimental molecular weight $(\mathrm{Mr})$ and isoelectric point $(\mathrm{p} I)$ matched for 8 of the 19 identified proteins. We used the ExPASy (Expert Protein Analysis System) proteomics server of the Swiss Institute of Bioinformatics for annotation and found that some of the identified proteins have a signal peptide. When the theoretical $\mathrm{p} I / \mathrm{Mr}$ of these proteins without the signal sequence was calculated, we found that another three spots (spots 6 , $53,74)$ matched the experimental and theoretical $\mathrm{pI} / \mathrm{Mr}$. The $\mathrm{p} I / \mathrm{Mr}$ discrepancy for the remaining spots might be due to the presence of different isoforms (spot 27), or to the amino acid sequence being derived from an EST that may not include the complete sequence (spots 66 and 77), or due to post-translational modification (spots 4 and 7).

\section{Discussion}

\subsection{Impacts of solar $U V-B$ and flavonoids on protein accumulation}

In this study, the accumulation of 67 peptide spots were affected by solar UV-B, with the total number of spots increasing in abundance being similar to the number of spots decreasing. Casati et al. (2005) found that 178 maize leaf protein spots were altered by UV-B radiation, and that more protein spots increased than decreased after UV-B exposure. This disparity may be due to the dissimilar species used, or to the different conditions used in the experiments. The maize experiments were conducted using both field and greenhouse conditions and the field experiments included both exclusion and supplement UV-B studies (Casati et al., 2005). In the present study, only UV-B exclusion was examined in the field.

Many more proteins were responsive to solar UV-B in the magenta line than in the standard line. Plants have evolved several mechanisms to cope with UV-B damage. One of the most important mechanisms is screening out UV-B radiation by accumulation of flavonoids in the leaf 
Table 1

Relative abundance of protein spots in different treatments

\begin{tabular}{|c|c|c|c|c|c|}
\hline \multirow[t]{3}{*}{ ID } & \multirow[t]{3}{*}{ Protein } & \multicolumn{4}{|c|}{ Relative abundance $^{\mathrm{A}}(\mathrm{Mean} \pm \mathrm{SD})$} \\
\hline & & \multicolumn{2}{|l|}{ Standard line } & \multicolumn{2}{|l|}{ Magenta line } \\
\hline & & With UV-B & No UV-B & With UV-B & No UV-B \\
\hline 1 & GCV P-protein & $0.310 \pm 0.03^{\mathrm{a}}$ & $0.256 \pm 0.02^{\mathrm{b}}$ & $0.247 \pm 0.01^{\mathrm{b}}$ & $0.239 \pm 0.01^{\mathrm{b}}$ \\
\hline 2 & Gamma-glutamyl hydrolase & $0.363 \pm 0.01^{\mathrm{a}}$ & $0.340 \pm 0.02^{\mathrm{a}}$ & $0.366 \pm 0.02^{\mathrm{a}}$ & $0.294 \pm 0.02^{\mathrm{b}}$ \\
\hline 3 & Rubisco activase & $0.440 \pm 0.02^{\mathrm{a}}$ & $0.361 \pm 0.03^{\mathrm{b}}$ & $0.434 \pm 0.04^{\mathrm{ab}}$ & $0.375 \pm 0.02^{\mathrm{b}}$ \\
\hline 6 & Triosephosphate isomerase Superoxide dismutase & $0.236 \pm 0.01^{\mathrm{a}}$ & $0.216 \pm 0.01^{\mathrm{a}}$ & $0.266 \pm 0.01^{\mathrm{b}}$ & $0.217 \pm 0.01^{\mathrm{a}}$ \\
\hline 7 & ATP synthase CF1 $60 \mathrm{kDa}$ chaperonin & $0.190 \pm 0.02^{\mathrm{a}}$ & $0.161 \pm 0.01^{\mathrm{a}}$ & $0.276 \pm 0.02^{\mathrm{b}}$ & $0.170 \pm 0.01^{\mathrm{a}}$ \\
\hline 8 & PSI subunit D & $1.411 \pm 0.04^{\mathrm{a}}$ & $1.401 \pm 0.06^{\mathrm{a}}$ & $1.346 \pm 0.06^{\mathrm{a}}$ & $0.933 \pm 0.05^{\mathrm{b}}$ \\
\hline 9 & PSI subunit IV A & $1.167 \pm 0.04^{\mathrm{a}}$ & $1.076 \pm 0.09^{\mathrm{a}}$ & $1.152 \pm 0.04^{\mathrm{a}}$ & $0.915 \pm 0.04^{\mathrm{b}}$ \\
\hline 10 & PSI PsaN subunit & $0.148 \pm 0.02^{\mathrm{a}}$ & $0.098 \pm 0.02^{\mathrm{b}}$ & $0.128 \pm 0.01^{\mathrm{ab}}$ & $0.090 \pm 0.01^{\mathrm{b}}$ \\
\hline 11 & PSI D2 subunit & $0.154 \pm 0.02^{\mathrm{a}}$ & $0.141 \pm 0.01^{\mathrm{a}}$ & $0.157 \pm 0.02^{\mathrm{a}}$ & $0.089 \pm 0.01^{\mathrm{b}}$ \\
\hline 17 & RNA-binding protein & $0.694 \pm 0.03^{\mathrm{a}}$ & $0.630 \pm 0.04^{\mathrm{b}}$ & $0.705 \pm 0.03^{\mathrm{a}}$ & $0.657 \pm 0.02^{\mathrm{ab}}$ \\
\hline 18 & $50 \mathrm{~S}$ ribosomal protein & $0.137 \pm 0.01^{\mathrm{a}}$ & $0.136 \pm 0.01^{\mathrm{a}}$ & $0.140 \pm 0.01^{\mathrm{a}}$ & $0.106 \pm 0.01^{\mathrm{b}}$ \\
\hline 19 & $30 \mathrm{~S}$ ribosomal protein & $0.235 \pm 0.01^{\mathrm{ab}}$ & $0.215 \pm 0.01^{\mathrm{a}}$ & $0.259 \pm 0.01^{\mathrm{b}}$ & $0.185 \pm 0.01^{\mathrm{c}}$ \\
\hline 20 & Vegetative storage protein & $1.042 \pm 0.04^{\mathrm{a}}$ & $0.921 \pm 0.13^{\mathrm{a}}$ & $1.045 \pm 0.05^{\mathrm{a}}$ & $0.713 \pm 0.02^{\mathrm{b}}$ \\
\hline 21 & Chaperonin 2 & $0.364 \pm 0.01^{\mathrm{ab}}$ & $0.346 \pm 0.01^{\mathrm{ac}}$ & $0.392 \pm 0.01^{\mathrm{b}}$ & $0.334 \pm 0.01^{\mathrm{c}}$ \\
\hline 22 & Cyclophilin & $0.280 \pm 0.02^{\mathrm{a}}$ & $0.242 \pm 0.03^{\mathrm{ab}}$ & $0.257 \pm 0.02^{\mathrm{a}}$ & $0.182 \pm 0.02^{\mathrm{b}}$ \\
\hline 23 & Vegetative storage protein & $0.530 \pm 0.02^{\mathrm{a}}$ & $0.467 \pm 0.06^{\mathrm{a}}$ & $0.465 \pm 0.04^{\mathrm{a}}$ & $0.353 \pm 0.03^{\mathrm{b}}$ \\
\hline 24 & Copper chaperone homolog & $0.094 \pm 0.01^{\mathrm{a}}$ & $0.072 \pm 0.01^{\mathrm{a}}$ & $0.100 \pm 0.01^{\mathrm{a}}$ & $0.052 \pm 0.01^{\mathrm{b}}$ \\
\hline 25 & $\mathrm{Cu}-\mathrm{Zn}$-superoxide dismutase & $0.089 \pm 0.01^{\mathrm{ab}}$ & $0.072 \pm 0.01^{\mathrm{a}}$ & $0.110 \pm 0.01^{\mathrm{b}}$ & $0.079 \pm 0.01^{\mathrm{a}}$ \\
\hline 26 & Ascorbate peroxidase & $0.421 \pm 0.01^{\mathrm{a}}$ & $0.434 \pm 0.02^{\mathrm{a}}$ & $0.439 \pm 0.01^{\mathrm{a}}$ & $0.351 \pm 0.01^{\mathrm{b}}$ \\
\hline 27 & Lectin & $0.318 \pm 0.02^{\mathrm{a}}$ & $0.246 \pm 0.03^{\mathrm{b}}$ & $0.297 \pm 0.02^{\mathrm{ab}}$ & $0.194 \pm 0.01^{\mathrm{c}}$ \\
\hline 28 & Putative protein & $0.160 \pm 0.01^{\mathrm{a}}$ & $0.132 \pm 0.03^{\mathrm{a}}$ & $0.138 \pm 0.01^{\mathrm{a}}$ & $0.093 \pm 0.01^{\mathrm{b}}$ \\
\hline 36 & SHMT & $0.511 \pm 0.03^{\mathrm{a}}$ & $0.526 \pm 0.01^{\mathrm{a}}$ & $0.510 \pm 0.02^{\mathrm{a}}$ & $0.586 \pm 0.03^{\mathrm{b}}$ \\
\hline 37 & SHMT & $0.224 \pm 0.01^{\mathrm{a}}$ & $0.216 \pm 0.02^{\mathrm{a}}$ & $0.272 \pm 0.01^{\mathrm{b}}$ & $0.315 \pm 0.01^{\mathrm{c}}$ \\
\hline 38 & Glutamine synthetase & $0.632 \pm 0.03^{\mathrm{a}}$ & $0.774 \pm 0.04^{\mathrm{b}}$ & $0.586 \pm 0.02^{\mathrm{a}}$ & $0.704 \pm 0.02^{\mathrm{c}}$ \\
\hline 39 & Glutamine synthetase & $0.185 \pm 0.01^{\mathrm{a}}$ & $0.221 \pm 0.02^{\mathrm{b}}$ & $0.155 \pm 0.01^{\mathrm{c}}$ & $0.193 \pm 0.01^{\mathrm{ab}}$ \\
\hline 40 & Phosphoglycerate kinase Glutamine synthetase & $0.145 \pm 0.03^{\mathrm{a}}$ & $0.238 \pm 0.03^{\mathrm{b}}$ & $0.132 \pm 0.01^{\mathrm{a}}$ & $0.206 \pm 0.01^{\mathrm{b}}$ \\
\hline 41 & Phosphoribulokinase & $0.460 \pm 0.02^{\mathrm{a}}$ & $0.466 \pm 0.03^{\mathrm{a}}$ & $0.384 \pm 0.02^{\mathrm{b}}$ & $0.434 \pm 0.01^{\mathrm{a}}$ \\
\hline 42 & Rubisco rbcS2 & $5.306 \pm 0.22^{\mathrm{a}}$ & $5.622 \pm 0.18^{\mathrm{a}}$ & $5.853 \pm 0.20^{\mathrm{a}}$ & $6.549 \pm 0.13^{\mathrm{b}}$ \\
\hline 43 & Rubisco rbcS4 & $0.280 \pm 0.01^{\mathrm{a}}$ & $0.306 \pm 0.01^{\mathrm{ab}}$ & $0.293 \pm 0.01^{\mathrm{a}}$ & $0.330 \pm 0.02^{\mathrm{b}}$ \\
\hline 44 & Rubisco activase & $0.142 \pm 0.02^{\mathrm{a}}$ & $0.233 \pm 0.01^{\mathrm{b}}$ & $0.136 \pm 0.01^{\mathrm{a}}$ & $0.189 \pm 0.01^{\mathrm{c}}$ \\
\hline 45 & Rubisco activase & $1.315 \pm 0.13^{\mathrm{a}}$ & $1.699 \pm 0.04^{\mathrm{b}}$ & $0.970 \pm 0.03^{\mathrm{c}}$ & $1.273 \pm 0.05^{\mathrm{a}}$ \\
\hline 46 & Rubisco activase & $0.050 \pm 0.01^{\mathrm{ab}}$ & $0.069 \pm 0.01^{\mathrm{ac}}$ & $0.047 \pm 0.01^{\mathrm{b}}$ & $0.071 \pm 0.01^{\mathrm{c}}$ \\
\hline 47 & Phosphoribulokinase & $0.444 \pm 0.04^{\mathrm{a}}$ & $0.676 \pm 0.02^{\mathrm{b}}$ & $0.429 \pm 0.01^{\mathrm{a}}$ & $0.643 \pm 0.02^{\mathrm{b}}$ \\
\hline 48 & GAPDH & $0.345 \pm 0.03^{\mathrm{a}}$ & $0.407 \pm 0.03^{\mathrm{ab}}$ & $0.356 \pm 0.02^{\mathrm{a}}$ & $0.431 \pm 0.02^{\mathrm{b}}$ \\
\hline 49 & GAPDH & $0.103 \pm 0.02^{\mathrm{a}}$ & $0.160 \pm 0.02^{\mathrm{b}}$ & $0.091 \pm 0.01^{\mathrm{a}}$ & $0.148 \pm 0.01^{\mathrm{b}}$ \\
\hline 50 & GAPDH & $0.117 \pm 0.02^{\mathrm{ab}}$ & $0.153 \pm 0.01^{\mathrm{ac}}$ & $0.108 \pm 0.01^{\mathrm{b}}$ & $0.159 \pm 0.01^{\mathrm{c}}$ \\
\hline 51 & GAPDH & $0.341 \pm 0.02^{\mathrm{a}}$ & $0.429 \pm 0.02^{\mathrm{b}}$ & $0.346 \pm 0.03^{\mathrm{a}}$ & $0.505 \pm 0.02^{\mathrm{c}}$ \\
\hline 52 & Phosphoglycerate kinase & $0.502 \pm 0.02^{\mathrm{a}}$ & $0.527 \pm 0.03^{\mathrm{a}}$ & $0.536 \pm 0.01^{\mathrm{a}}$ & $0.619 \pm 0.02^{\mathrm{b}}$ \\
\hline 53 & Ferredoxin NADP reductase & $0.165 \pm 0.01^{\mathrm{a}}$ & $0.204 \pm 0.01^{\mathrm{b}}$ & $0.170 \pm 0.01^{\mathrm{a}}$ & $0.176 \pm 0.01^{\mathrm{a}}$ \\
\hline 54 & Carbonic anhydrase & $0.679 \pm 0.04$ & $0.695 \pm 0.04^{\mathrm{a}}$ & $0.640 \pm 0.05^{\mathrm{a}}$ & $0.832 \pm 0.05^{\mathrm{b}}$ \\
\hline 55 & Carbonic anhydrase & $0.298 \pm 0.02^{\mathrm{ab}}$ & $0.330 \pm 0.02^{\mathrm{a}}$ & $0.269 \pm 0.02^{\mathrm{b}}$ & $0.384 \pm 0.018^{\mathrm{ac}}$ \\
\hline 56 & Translation elongation factor & $0.133 \pm 0.01^{\mathrm{a}}$ & $0.178 \pm 0.01^{\mathrm{b}}$ & $0.124 \pm 0.02$ & $0.202 \pm 0.01^{\mathrm{c}}$ \\
\hline 57 & Chaperonin precursor & $0.179 \pm 0.01^{\mathrm{a}}$ & $0.214 \pm 0.01^{\mathrm{b}}$ & $0.170 \pm 0.01^{\mathrm{a}}$ & $0.231 \pm 0.01^{\mathrm{b}}$ \\
\hline
\end{tabular}


Table 1 (continued)

\begin{tabular}{|c|c|c|c|c|c|}
\hline \multirow[t]{3}{*}{ ID } & \multirow[t]{3}{*}{ Protein } & \multicolumn{4}{|c|}{ Relative abundance $^{\mathrm{A}}($ Mean $\pm \mathrm{SD})$} \\
\hline & & \multicolumn{2}{|l|}{ Standard line } & \multicolumn{2}{|l|}{ Magenta line } \\
\hline & & With UV-B & No UV-B & With UV-B & No UV-B \\
\hline 59 & Chaperonin precursor & $0.121 \pm 0.01^{\mathrm{a}}$ & $0.161 \pm 0.01^{\mathrm{b}}$ & $0.095 \pm 0.01^{\mathrm{c}}$ & $0.153 \pm 0.01^{\mathrm{b}}$ \\
\hline 60 & ER HSC70-cognate binding protein & $0.350 \pm 0.02^{\mathrm{a}}$ & $0.383 \pm 0.02^{\mathrm{a}}$ & $0.298 \pm 0.02^{\mathrm{b}}$ & $0.361 \pm 0.02^{\mathrm{a}}$ \\
\hline 61 & ER HSC70-cognate binding protein & $0.374 \pm 0.01^{\mathrm{a}}$ & $0.466 \pm 0.01^{\mathrm{b}}$ & $0.337 \pm 0.01^{\mathrm{c}}$ & $0.428 \pm 0.01^{\mathrm{d}}$ \\
\hline 64 & Peroxiredoxin & $0.232 \pm 0.03^{\mathrm{a}}$ & $0.246 \pm 0.01^{\mathrm{a}}$ & $0.240 \pm 0.02^{\mathrm{a}}$ & $0.307 \pm 0.01^{\mathrm{b}}$ \\
\hline 65 & 1-Deoxy-D-xylulose 5-phosphate reductoisomerase & $0.277 \pm 0.01^{\mathrm{a}}$ & $0.269 \pm 0.02^{\mathrm{a}}$ & $0.234 \pm 0.01^{\mathrm{b}}$ & $0.298 \pm 0.01^{\mathrm{a}}$ \\
\hline 66 & Chalcone reductase & $0.106 \pm 0.01^{\mathrm{a}}$ & $0.120 \pm 0.01^{\mathrm{a}}$ & $0.087 \pm 0.01^{\mathrm{b}}$ & $0.110 \pm 0.01^{\mathrm{a}}$ \\
\hline 67 & Unidentified & $0.074 \pm 0.01^{\mathrm{a}}$ & $0.095 \pm 0.01^{\mathrm{b}}$ & $0.069 \pm 0.01^{\mathrm{a}}$ & $0.063 \pm 0.01^{\mathrm{a}}$ \\
\hline \multicolumn{6}{|c|}{ Group III: Spots not altered by $U V-B$ but had different accumulations between the two lines } \\
\hline 68 & GCV H-protein & $0.223 \pm 0.03^{\mathrm{ab}}$ & $0.266 \pm 0.02^{\mathrm{a}}$ & $0.200 \pm 0.03^{\mathrm{b}}$ & $0.193 \pm 0.01^{\mathrm{b}}$ \\
\hline 73 & Carbonic anhydrase & $0.576 \pm 0.03^{\mathrm{a}}$ & $0.527 \pm 0.04^{\mathrm{a}}$ & $0.458 \pm 0.02^{\mathrm{b}}$ & $0.450 \pm 0.02^{\mathrm{b}}$ \\
\hline 74 & Carbonic anhydrase & $0.173 \pm 0.01^{\mathrm{a}}$ & $0.167 \pm 0.02^{\mathrm{a}}$ & $0.127 \pm 0.02^{\mathrm{b}}$ & $0.107 \pm 0.01^{\mathrm{b}}$ \\
\hline 75 & Heat shock protein 70 & $0.819 \pm 0.04^{\mathrm{a}}$ & $0.839 \pm 0.02^{\mathrm{a}}$ & $0.872 \pm 0.04^{\mathrm{ab}}$ & $0.976 \pm 0.05^{\mathrm{b}}$ \\
\hline 76 & PR1 A precursor & $0.133 \pm 0.01^{\mathrm{ab}}$ & $0.187 \pm 0.02^{\mathrm{a}}$ & $0.115 \pm 0.02^{\mathrm{bc}}$ & $0.084 \pm 0.01^{\mathrm{c}}$ \\
\hline 77 & Thaumatin-like protein & $0.146 \pm 0.01^{\mathrm{a}}$ & $0.192 \pm 0.03^{\mathrm{a}}$ & $0.306 \pm 0.03^{\mathrm{b}}$ & $0.285 \pm 0.02^{\mathrm{b}}$ \\
\hline 78 & Unidentified & $0.108 \pm 0.01^{\mathrm{a}}$ & $0.120 \pm 0.01^{\mathrm{a}}$ & $0.074 \pm 0.01^{\mathrm{b}}$ & $0.074 \pm 0.01^{\mathrm{b}}$ \\
\hline 79 & Unidentified & $0.082 \pm 0.01^{\mathrm{ab}}$ & $0.086 \pm 0.01^{\mathrm{a}}$ & $0.061 \pm 0.01^{\mathrm{b}}$ & $0.041 \pm 0.01^{\mathrm{b}}$ \\
\hline
\end{tabular}

${ }^{\mathrm{A}}$ Different letters indicate significantly different means separated by least significant difference $(P \leqslant 0.05)$. Spot numbers (ID) correspond to Fig. 1.

Table 2

Proteins identified from the soybean leaf by MS

\begin{tabular}{|c|c|c|c|c|c|c|}
\hline ID & Protein identification [species] & $\mathrm{T} \mathrm{Mr} / \mathrm{p} I$ & MO & PM & $\mathrm{SC}$ & Acce. No. \\
\hline 1 & GCV P-protein [Pisum sativum] & $115411 / 7.17$ & 73 & 2 & 5 & gi|20741 \\
\hline 3 & Rubisco activase [Vigna radiata] & $48042 / 7.57$ & 75 & 10 & 27 & gi|8954287 \\
\hline 4 & Rubisco large subunit [Tribeles australis] & $52050 / 6.13$ & 132 & 5 & 11 & gi|9910002 \\
\hline \multirow[t]{2}{*}{6} & Triosephosphate isomerase $[$ Fragaria $\times$ ananassa $]$ & $33733 / 7.64$ & 496 & 17 & 35 & gi|7650502 \\
\hline & Superoxide dismutase [Glycine $\max ]$ & $27881 / 5.60$ & 308 & 11 & 27 & gi|134646 \\
\hline \multirow[t]{2}{*}{7} & ATP synthase CF1 epsilon sub [G. max $]$ & $14801 / 5.41$ & 291 & 13 & 41 & gi|91214127 \\
\hline & $60 \mathrm{kDa}$ chaperonin [Ricinus communis] & $52461 / 4.77$ & 186 & 5 & 9 & gi|134101 \\
\hline 24 & Copper chaperone homolog $[G . \max ]$ & $13753 / 4.66$ & 92 & 3 & 20 & gi|6525011 \\
\hline 25 & $\mathrm{Cu}-\mathrm{Zn}$-superoxide dismutase $[$ Pinus pinaster $]$ & $22328 / 6.11$ & 103 & 2 & 13 & gi|16798638 \\
\hline 27 & Lectin $[G . \max ]$ & $22568 / 9.59$ & 155 & 4 & 18 & gi|81238245 \\
\hline 28 & Putative protein [Arabidopsis thaliana] & $27880 / 8.31$ & 93 & 3 & 10 & gi|7269238 \\
\hline 41 & Phosphoribulokinase $[P$. sativum $]$ & $39230 / 5.41$ & 471 & 14 & 32 & gi|1885326 \\
\hline 46 & Rubisco activase [Phaseolus vulgaris] & $48342 / 8.19$ & 142 & 4 & 10 & gi|3033513 \\
\hline 51 & GAPDH $[G . \max ]$ & $43479 / 8.42$ & 668 & 20 & 35 & gi| 77540210 \\
\hline 53 & Ferredoxin NADP reductase $[$ A. thaliana $]$ & $40643 / 8.32$ & 171 & 5 & 16 & gi|10177134 \\
\hline 62 & Metalloproteinase $[G . \max ]$ & $19001 / 5.82$ & 135 & 5 & 19 & gi|384337 \\
\hline 66 & Chalcone reductase $[G . \max ]$ & $23143 / 8.63$ & 81 & 2 & 10 & gi|7588574 \\
\hline 74 & Carbonic anhydrase [Vigna radiata] & $35804 / 7.59$ & 66 & 2 & 6 & gi|8954289 \\
\hline 77 & Thaumatin-like protein precursor [G. $\max ]$ & $17425 / 4.12$ & 105 & 2 & 19 & gi|15000431 \\
\hline
\end{tabular}

All spots were identified by LC-MS/MS except spot 3, which was identified by MALDI-TOF. The NCBI nr database was searched for all the spots except spots 66 and 77 for which the EST_others database was searched. ID: Spot number (Fig. 1); T Mr/pI: Theoretical Mr/pI; MO: MOWSE score; PM: Number of peptides matched; SC: Percentage of sequence coverage (\%); Acce. No.: Accession number.

epidermis (Schmelzer et al., 1988; Robberecht and Caldwell, 1983). The increased response of the magenta line to UV-B may be related to its reduced flavonoid content. Of the group III peptides, 11 spots (spots 68-70, 72-79) showed different intensities between the two lines even without UV-B treatment (Table 1). This might be due to the high UV-A and PAR levels in the solar radiation and/or oxidative stress caused by other stress factors such as high temperature. While flavonoids provide UV-B protection by absorbing radiation in UV-B region, they also 
are effective UV-A absorbers. Although it is less damaging on a photon basis than UV-B, UV-A comprises a much larger portion of the solar radiation than does UV-B, and UV-A is able to penetrate to greater depths within the leaf than UV-B (Liakoura et al., 2003). The greater responses under UV-B exclusion in the magenta line could be related to its inability to synthesize flavonoids. Irradiation of the magenta line with solar UV radiation could have resulted in increased flux of UV-A through the more transparent epidermis reaching mesophyll cells, as compared to the standard line capable of synthesizing flavonoids. Also, because flavonoids can act as antioxidants (Peng et al., 2003), their absence in the magenta line could also lead to greater oxidative stress, and this hypothesis is supported by evidence for greater oxidative responses in the magenta line (data not shown).

Many proteins are represented by multiple spots (Casati et al., 2005; Sarnighausen et al., 2004; Giavalisco et al., 2005). The multiple spots may be different products of closely related genes or PTM of a single protein. Taking into account the multiplicity of spots, we detected 47 unique proteins altered by UV-B radiation (Table 1). In maize, Casati et al. (2005) reported that 14 proteins that were represented with multiple spots showed opposite effect by UV-B radiation. For example, three spots of the Rubisco large subunit were increased while another two spots of Rubisco large subunit were decreased by UV-B radiation (Casati et al., 2005). Similar results were observed for Rubisco activase and glycine cleavage system (GCV) P-protein in the present study. One spot (spot 3) of Rubisco activase was increased by UV-B, while another three spots (spots 44-46) of this protein were decreased. In the present study, most of the spots contained only one protein, but three spots contained two different proteins (spot 6: triosephosphate isomerase and superoxide dismutase; spot 7: ATP synthase CF1 epsilon subunit and $60 \mathrm{kDa}$ chaperonin alpha subunit; spot 40: phosphoglycerate kinase and glutamine synthetase). These spots were altered by solar UV-B. UV-B may affect one or both of the proteins in each spot, although at this stage of our investigation we could not distinguish between these possibilities.

\subsection{Functional analyses of proteins responsive to $U V-B$}

The identified proteins in this study were classified according to the functional categories described by Bevan et al. (1998) (Table 3). The proteins are quite diverse and are involved in metabolism, energy, protein destination/ storage, disease/defense, transcription, protein synthesis, and secondary metabolism. The functional group with the largest number of protein spot altered by UV-B was the energy category. In this category, 30 protein spots were altered by UV-B, 14 increased while 16 decreased (Table 1 and 3). Nine spots were identified as photosystem (PS) II oxygen-evolving enhancer (OEE) (spots 12-16) or subunits of PS I (spots 8-11), and all were enhanced by solar UV-B. Only one protein spot (spot 7: epsilon subunit of ATP synthase CF1) is involved in electron transport, and this spot was also increased by UV-B. Using microarray technology, Izaguirre et al. (2003) found that most genes encoding PS polypeptides were decreased by solar UV-B, but one gene, encoding the PS II OEE $23-\mathrm{kDa}$ polypeptide, was increased. Interestingly, all the responsive proteins related to PS were enhanced by solar UV-B in the present study.

The other spots affected by UV-B in the energy category were identified as enzymes involved in primary carbon metabolism. Most of the spots, identified as Rubisco activase (spots 44-46), Rubisco small subunit (spots 42 and 43), glyceraldehyde-3-phosphate dehydrogenase (GAPDH) (spots 48-51), phosphoglycerate kinase (spots 40 and 52), phosphoribulokinase (spots 41 and 47) or other enzymes (spots 53-55), were decreased by UV-B radiation (Tables 1 and 3). In addition to catalyzing reactions in the Calvin cycle, GAPDH is also reported to have protein kinase activity (Duclos-Vallee et al., 1998), to bind RNA (Nagy and Rigby, 1995), and to increase ribozyme (Sioud and Jespersen, 1996) and phosphotransferase activities (Engel et al., 1998). Other environmental stresses have been reported to increase GAPDH level (Yang et al., 1993; Chang et al., 2000; Russell et al., 1990), however, four spots of GAPDH were decreased by UV-B in the present study. This inconsistency may be due to the multiple isoforms of GAPDH. Izaguirre et al. (2003) also found that some genes for Calvin cycle enzymes were decreased by

Table 3

Functional distribution of protein spots responsive to UV-B

\begin{tabular}{|c|c|c|c|c|c|c|c|c|c|}
\hline \multirow{2}{*}{$\begin{array}{l}\text { UV-B } \\
\text { effects }\end{array}$} & \multirow[t]{2}{*}{ Lines } & \multicolumn{8}{|l|}{ Protein spot number ${ }^{\mathrm{a}}$} \\
\hline & & Energy & Metabolism & $\begin{array}{l}\text { Protein } \\
\text { destination } \\
\text { storage }\end{array}$ & Transcription & $\begin{array}{l}\text { Protein } \\
\text { synthesis }\end{array}$ & $\begin{array}{l}\text { Disease } \\
\text { defence }\end{array}$ & $\begin{array}{l}\text { Secondary } \\
\text { metabolism }\end{array}$ & Unclear \\
\hline \multirow[t]{2}{*}{ Increase } & Standard & $3,5,10$ & 1 & & 17 & & 27 & & 29,30 \\
\hline & Magenta & $4-9,11-16$ & 2 & $7,20-24$ & & 18,19 & $6,25-27$ & & $28-31$ \\
\hline \multirow[t]{2}{*}{ Decrease } & Standard & $40,44,45,47,49,51,53$ & $\begin{array}{l}32-34,38- \\
40\end{array}$ & $57-59,61,62$ & & 56 & 63 & & 67 \\
\hline & Magenta & $40-52,54,55$ & $32,35-40$ & $57-61$ & & 56 & 64 & 65,66 & \\
\hline
\end{tabular}

Protein spots were grouped according to the functional categories described by Bevan et al. (1998).

${ }^{a}$ Spot numbers correspond to Fig. 1. 
solar UV-B. It is reported that the inhibition of UV-B on photosynthesis is associated with enzymatic, rather than PS II limitations (Xiong and Day, 2001). Sullivan and Teramura (1990) found that UV-induced reductions involving PS in soybean occurred only at high internal $\mathrm{CO}_{2}$ levels, suggesting that UV-B could lead to increased substrate limitations on photosynthesis. Also, some studies indicated that UV-B could reduce the carbohydrate levels in plants (Quaggiotti et al., 2004; Ghisi et al., 2002). Consistent with these results, the enzymes involved in $\mathrm{CO}_{2}$ assimilation were suppressed by solar UV-B in this study. The remaining spots (spot 3: Rubisco activase; spot 4: Rubisco large subunit; spot 5: ribulose-phosphate 3-epimerase; spot 6: triosephosphate isomerase) were increased by UV-B. Spot 4 is only a fragment of Rubisco large subunit because its sequence only matches the first half part of Rubisco and it has very low Mr. Therefore, an increase in spot 4 most probably indicates increased degradation of the Rubisco large subunit.

Increases in proteins related to PS and electron transport may lead to greater reducing power. However, ambient levels of UV-B may limit $\mathrm{CO}_{2}$ fixation and reduce the regeneration of $\mathrm{NADP}^{+}$and ribulose bisphosphate, because the enzymes involved in the Calvin cycle were decreased. For example, GAPDH and phosphoglycerate kinase both are key enzymes in the regeneration of ribulose bisphosphate and reducing power. Therefore, the photosynthetic electron transport chain may be over reduced, leading to the formation of superoxide radicals and singlet oxygen. $\mathrm{Xu}$ et al. (data not shown) have found that solar UV-B radiation does cause oxidative stress in the two soybean lines.

The metabolism category includes 11 spots altered by UV-B (Table 3). Two of them (spot 1: GCV T- protein; spot 2: gamma-glutamyl hydrolase) increased in abundance, while 9 spots, identified as glutamine synthetase (spots 38-40), alanine aminotransferase (spot 35), GCV T- or P-protein (spots 32-34), and serine hydroxymethyltransferase (SHMT) (spots 36 and 37), were decreased by solar UV-B. These enzymes are involved in nitrogen metabolism. Glutamine synthetase catalyzes the assimilation of ammonium to glutamine using glutamic acid as its substrate (Chen and Silflow, 1996). Reduction of this enzyme under stress conditions has been reported, and this may be a protective mechanism because nitric oxide, an intermediate of nitrogen assimilation, is an active radical (Wang et al., 2004). It has been reported that UV-B can decrease nitrogen assimilation in some plants (Quaggiotti et al., 2004; Ghisi et al., 2002; Balakumar et al., 1999). The reduction of enzymes involved in primary nitrogen and carbon metabolism indicates redirection of carbon and nitrogen resources into other pathways, such as those involved in repair or protection processes.

The protein destination and storage category includes 12 spots altered by solar UV-B (Table 3). Several spots (spots 57-61) of chaperonin and associated co-chaperones were decreased by solar UV-B. Other spots, identified as $60 \mathrm{kDa}$ chaperonin (spot 7), vegetative storage protein (spots 20 and 23), chaperonin 2 (spot 21), copper chaperone homolog (spot 24), and cyclophilin (spot 22), were increased by UV-B radiation. Chaperones are proteins whose function is to assist other proteins in achieving proper folding, and have been shown to accumulate in plants in response to many stresses (Wang et al., 2004; Yan et al., 2006). In this study, some chaperone spots (spots 7,21,24) were enhanced while others (spots 57-61) were decreased by UV-B, and similar results were observed under UV-B stress by Casati et al. (2005). These results can be explained by the varied functions of chaperones. Some chaperones act to repair the potential damage caused by misfolding; some are involved in folding newly made proteins as they are extruded from the ribosome; and others are involved in transport across membranes (Georgopoulos and Welch, 1993; Leone et al., 2000).

Only six protein spots involved in the disease and defense category were altered by UV-B radiation (Table 2). Two of them, identified as catalase (spot 63) and peroxiredoxin (spot 64), were decreased by UV-B. Four spots, identified as ascorbate peroxidase (spot 26), superoxide dismutase (spots 6 and 25), and lectin (spot 27), were increased by UV-B. All six proteins, except the lectin, are enzymes that destroy active oxygen species and are usually increased under stress conditions (Wang et al., 2004; Yan et al., 2006). Under the same conditions, solar UV-B increased the total activity of ascorbate peroxidase, decreased the total activity of superoxide dismutase, and had no effect on catalase activity (Xu et al., data not shown). However, total enzymatic activity does not reflect the changes in the different isoforms of the same enzyme, and enzyme activity could be altered without changes in protein quantity.

Only two spots in the secondary metabolism category (spot 65: 1-deoxy-D-xylulose 5-phosphate reductoisomerase; spot 66: chalcone reductase) were responsive to solar UV-B (Table 3). These two spots were decreased by solar UV-B in the magenta line but not in the standard line. Increases in leaf flavonoids concentration are the most consistent response to supplemental UV-B (Searles et al., 2001). However, increases in proteins involved in the flavonoid biosynthetic pathway were not detected in the present study. However, proteins involved in flavonoid synthesis may be in too low abundance to be detected on these gels, because more than one hundred protein spots were identified and no other proteins associated with secondary metabolism were found. The one spot (spot 17) responsive to solar UV-B in the transcription category was identified as RNA-binding protein, and its abundance was increased by UV-B. The protein synthesis category has three spots regulated by solar UV-B. Two of them (spot 18: 50S ribosomal protein; spot 19: 30S ribosomal protein) were increased in abundance in response to UV-B, while spot 56 , translation elongation factor, decreased in abundance.

In summary, our results indicate that proteins related to the photosynthetic photosystems increased in abundance, while enzymes involved in primary carbon and nitrogen 
metabolism decreased, and substantiate that flavonoids act as screening compounds in protecting plants from UV-B radiation. No effects on proteins involved in the signal transduction were detected, possibly because many of the proteins involved in the signal transduction occur in too low abundance to be detected in crude extracts, or because membrane proteins are usually under-represented on 2-D PAGE gels. This study provides new insights into the responses in the soybean leaf to solar UV-B radiation. Further studies are needed to better understand the molecular basis of the UV-B response in soybean.

\section{Materials and methods}

\subsection{Plant materials and experiment design}

Seeds of the standard and magenta isolines of the Clark cultivar of soybean were planted in pots in a greenhouse at the University of Maryland (College Park, MD) and allowed to germinate for 3 days. Following this period the plants were moved to the USDA Southfarm (Beltsville, MD) where they were separated into two UV-B treatment regimes. Half of the plants were placed inside either of two open-ended exclusion shelters made of polyester (DuPont, Circleville, OH, USA), which absorbs almost all solar radiation below $316 \mathrm{~nm}$. The remaining plants were placed under another two shelters covered by clear Teflon (DuPont, Circleville, OH, USA), which is virtually transparent to solar UV radiation. The materials are similar in transmission properties in the UV-A and PAR wavelength. The plants beneath the polyester filters received very little UV-B radiation and served as controls for seasonal changes in temperature and PAR, etc. Plants were rotated every day and watered to minimize the occurrence of drought stress. The first trifoliates appeared on July 4th, 2005 and were harvested from 3 to 5 plants for each sample when they were 12-day old. Five independent samples were harvested for each treatment replicate. The harvested samples were immediately frozen in liquid nitrogen, and then stored at $-80^{\circ} \mathrm{C}$ prior to analysis.

\subsection{Protein extraction and 2-D PAGE}

Frozen samples were ground with liquid nitrogen and incubated with $10 \%$ trichloroacetic acid (TCA) and $0.07 \% 2$-mercaptoethanol in acetone for $1 \mathrm{~h}$ at $-20{ }^{\circ} \mathrm{C}$. The precipitated proteins were pelleted and washed with ice-cold acetone containing $0.07 \%$ 2-mercaptoethanol to remove pigments and lipids until the supernatant was colorless. The pellet was vacuum dried, resuspended in resolubilization solution ( $9 \mathrm{M}$ urea, $1 \%$ CHAPS, $1 \%$ DTT, $1 \%$ pharmalyte) and sonicated to extract proteins. Insoluble tissue was removed by centrifugation at $21,000 \mathrm{~g}$ for 20 min. Protein concentration was determined according to Bradford (1976) using a commercial dye reagent (BioRad Laboratories, Hercules, CA) with BSA as a standard.
An IPGPhor apparatus (GE Healthcare, Piscataway, NJ) was used for isoelectric focusing (IEF) with immobilized $\mathrm{pH}$ gradient (IPG) strips ( $\mathrm{pH} 3.0-10.0$, linear gradient, $13 \mathrm{~cm}$ ). The IPG strips were rehydrated for $12 \mathrm{~h}$ with $250 \mu \mathrm{L}$ rehydration buffer ( $8 \mathrm{M}$ urea, $2 \%$ CHAPS, $0.5 \%$ pharmalyte, $0.002 \%$ bromophenol blue) containing $350 \mu \mathrm{g}$ proteins. The voltage settings for IEF were $500 \mathrm{~V}$ for $1 \mathrm{~h}$, $1000 \mathrm{~V}$ for $1 \mathrm{~h}, 5000 \mathrm{~V}$ for $1 \mathrm{~h}$, and $8000 \mathrm{~V}$ to a total $46.86 \mathrm{kVh}$. Following electrophoresis, the protein in the strips was denatured with equilibration buffer $(50 \mathrm{mM}$ Tris- $\mathrm{HCl}$ pH 8.8, $6 \mathrm{M}$ urea, $30 \%$ glycerol, $2 \%$ SDS, $0.002 \%$ bromophenol blue, $1 \%$ DTT) and then incubated with the same buffer containing $2.5 \%$ iodoacetamide instead of DTT for $30 \mathrm{~min}$ at room temperature. The second dimension electrophoresis was performed on a $12.5 \%$ gel using a Hoefer SE 600 Ruby electrophoresis unit (GE Healthcare, Piscataway, NJ). The gels were stained with Coomassie brilliant blue (CBB) G-250 (Newsholme et al., 2000) and scanned using a Personal Densitometer SI (GE Healthcare, Piscataway, NJ).

\subsection{Image acquisition and data analysis}

Densitometry images were analyzed with Image Master 2D-Elite (version 4.01) (GE Healthcare, Piscataway, NJ) software. Image analysis included the following procedures: spot detection, spot measurement, background subtraction, and spot matching. Only spots that were detected on all the five replicate gels were further analyzed. To correct the variability due to CBB staining, the spot volumes were normalized as a percentage of the total volume of all spots on the gel. Data were subjected to analysis of variance to test for the effects of cultivar, UV-B and their interactions. Significantly different means were separated by least significant difference $(P<0.05)$ using SAS software (1995).

\subsection{Spots digestion and $M S$}

Protein digestion was performed as described previously (Natarajan et al., 2005). Gel spots were washed with $\mathrm{CH}_{3} \mathrm{CN}: \mathrm{H}_{2} \mathrm{O}(1: 1, \mathrm{v} / \mathrm{v})$ containing $25 \mathrm{mM}$ ammonium bicarbonate to remove the dye, dehydrated with $100 \%$ $\mathrm{ACN}$, dried under vacuum, and incubated overnight at $37^{\circ} \mathrm{C}$ with $20 \mu \mathrm{L}$ of $10 \mu \mathrm{g} / \mathrm{mL}$ trypsin (modified porcine trypsin, sequencing grade, Promega, Madison, WI) in $20 \mathrm{mM}$ ammonium bicarbonate. The resulting tryptic fragments were eluted by diffusion into $\mathrm{CH}_{3} \mathrm{CN}: \mathrm{H}_{2} \mathrm{O}(1: 1$, v/v) and $0.5 \%$ trifluoroacetic acid (TFA). The extract was vacuum dried and the pellet was dissolved in $\mathrm{CH}_{3} \mathrm{CN}: \mathrm{H}_{2} \mathrm{O}$ $(1: 1, \mathrm{v} / \mathrm{v})$ and $0.1 \%$ TFA.

For peptide mass fingerprinting (PMF), a Voyager DESTR MALDI-TOF mass spectrometer (Applied Biosystems, Framingham, MA) operated in positive ion reflector mode was used to analyze tryptic peptides. Samples were co-crystallized with $\alpha$-cyanohydroxycinnamic acid matrix, and spectra were acquired with 50 shots of a $337 \mathrm{~nm}$ 
Nitrogen Laser operating at $20 \mathrm{~Hz}$. Spectra were calibrated using the trypsin autolysis peaks at $\mathrm{m} / \mathrm{z} 842.51$ and 2,211.10 as internal standards. For LC-MS/MS a Thermo Finnigan LCQ Deca XP plus Ion Trap mass spectrometer was used to analyze proteins. Peptides were separated on a reverse phase column using a $30 \mathrm{~min}$ gradient of $5-60 \% \mathrm{ACN}$ in water with $0.1 \%$ formic acid. The instrument was operated with a duty cycle that acquired MS/MS spectra on the three most abundant ions identified by a survey scan from 300 to $2000 \mathrm{Da}$. Dynamic exclusion was employed to prevent the continuous analysis of the same ions. Once two MS/MS spectra of any given ion had been acquired, the parent mass was placed on an exclusion list for the duration of $1.5 \mathrm{~min}$. The raw data were processed by Sequest to generate DTA files for database searching. The merge.pl script from Matrix Science was used to convert multiple Sequest DTA files into a single mascot generic file suitable for searching in Mascot (Perkins et al., 1999).

\subsection{Protein identification}

Protein identification was performed using the Mascot search engine, which uses a probability based scoring system (Perkins et al., 1999). The NCBI nr database was selected as the primary database to be searched. For LCMS/MS, if the primary database did not yield identity, the "EST_others" database was queried. The following parameters were used for database searches with MALDI-TOF PMF data: monoisotopic mass, $25 \mathrm{ppm}$ mass accuracy, trypsin as digesting enzyme with 1 missed cleavage allowed, carbamidomethylation of cysteine as a fixed modification, oxidation of methionine, N-terminal pyroglutamic acid from glutamic acid or glutamine as allowable variable modifications. For database searches with MS/MS spectra, the following parameters were used: monoisotopic mass; 1.5 Da peptide and MS/MS mass tolerance; peptide charge of $+1,+2$, or +3 ; trypsin as digesting enzyme with 1 missed cleavage allowed; carbamidomethylation of cysteine as a fixed modification; oxidation of methionine, N-terminal pyroglutamic acid from glutamic acid or glutamine as allowable variable modifications. Taxonomy was limited to green plants for both MALDI and MS/MS ion searches. For MALDITOF MS data to qualify as a positive identification a protein's score had to equal or exceed the minimum significant score of 64 . Positive identifications of proteins by MS/MS analysis required a minimum of two unique peptides, with at least one peptide having a significant ion score.

\section{Acknowledgements}

This work was supported in part by the USDA UV-B Monitoring and Research Program and the University of Maryland Agricultural Experiment Station. The authors thank Mr. Mirecki, Mr. DeStefano and Ms. Pope for assistance with UV exclusion shelters. The authors also thank Dr. W. Kenworthy for providing soybean seeds.

\section{References}

A-H-Mackerness, S., John, C.F., Jordan, B., Thomas, B., 2001. Early signaling components in ultraviolet-B response: distinct roles for different reactive oxygen species and nitric oxide. FEBS Lett. 489, $237-$ 242.

A-H-Mackerness, S., Surplus, S.L., Blake, P., John, C.F., BuchananWollaston, V., Jordan, B.R., Thomas, B., 1999. Ultraviolet-B-induced stress and changes in gene expression in Arabidopsis thaliana: role of signaling pathways controlled by jasmonic acid, ethylene and reactive oxygen species. Plant Cell Environ. 22, 1413-1423.

A-H-Mackerness, S., Surplus, S.L., Jordan, B.R., Thomas, B., 1998 Effects of supplementary ultraviolet-B radiation on photosynthetic transcripts at different stages of leaf development and light levels in pea (Pisum sativum L.): role of active oxygen species and antioxidant enzymes. Photochem. Photobiol. 68, 88-96.

A-H-Mackerness, S., Thomas, B., Jordan, B.R., 1997. The effect of supplementary ultraviolet-B radiation on mRNA transcripts, translation and stability of chloroplast proteins and pigment formation in Pisum sativum L. Exp. Bot. 48, 729-738.

Alves, M., Francisco, R., Martins, I., Ricardo, C.P.P., 2006. Analysis of lupinus albus leaf apoplastic proteins in response to boron deficiency. Plant Soil 279, 1-11.

Balakumar, T., Selvakumar, V., Sathiameena, K., Ilanchezhian, C.M., Paliwal, K., 1999. UV-B radiation mediated alterations in the nitrate assimilation pathway of crop plants 1 . Kinetic characteristics of nitrate reductase. Photosynthetica 37, 459-467.

Ballare, C.L., Barnes, P.W., Flint, S.D., 1995. Inhibition of hypocotyl elongation by ultraviolet-B radiation in de-etiolating tomato seedlings. I. The photoreceptor. Physiol. Plant 93, 584-592.

Bevan, M., Bancroft, I., Bent, E., Love, K., Goodman, H., Dean, C., Bergkamp, R., Dirske, W., Van Staveren, M., Stiekema, W., 1998. Analysis of $1.9 \mathrm{Mb}$ of contiguous sequence from chromosome 4 of Arabidopsis thaliana. Nature 391, 485-488.

Blumthaler, M., Amback, W., 1990. Induction of increasing solar ultraviolet-B radiation flux in Alpine regions. Science 248, 206-208.

Bradford, M.M., 1976. A rapid and sensitive method for the quantitation of microgram quantities of protein utilizing the principle of proteindye binding. Anal. Biochem. 72, 248-254.

Bray, C.M., West, C.E., 2005. DNA repair mechanisms in plants: crucial sensors and effectors for the maintenance of genome integrity. New Phytol. 168, 511-528.

Buzzell, R.I., Buttery, B.R., Bernard, R.I., 1977. Inheritance and linkage of a magenta flower gene in soybeans. Can. J. Genet. Cytol. 19, 749 751.

Casati, P., Walbot, V., 2003. Gene expression profiling in response to ultraviolet radiation in Zea mays genotypes with varying flavonoid content. Plant Physiol. 132, 1739-1754.

Casati, P., Walbot, V., 2004. Rapid transcriptome responses of maize (Zea mays) to UV-B in irradiated and shielded tissues. Genome Biol. 5, R16.

Casati, P., Zhang, X., Burlingame, A.L., Walbot, V., 2005. Analysis of leaf proteome after UV-B irradiation in maize lines differing in sensitivity. Mol. Cell. Proteomics 4, 1673-1685.

Castro, A.J., Carapito, C., Zorn, N., Magné, C., Leize, E., Dorsselaer, A.V., Clément, C., 2005. Proteomic analysis of grapevine (Vitis vinifera L.) tissues subjected to herbicide stress. J Exp. Bot. 56, 2783-2795.

Chang, W.W.P., Huang, L., Shen, M., Webster, C., Burlingame, A.L., Roberts, J.K.M., 2000. Patterns of protein synthesis and tolerance of anoxia in root tips of maize seedlings acclimated to a low-oxygen environment, and identification of proteins by mass spectrometry. Plant Physiol. 122, 295-317.

Chappell, J., Hahlbrock, K., 1984. Transcription of plant defence genes in response to UV light or fungal elicitor. Nature 311, 76-78. 
Chen, Q., Silflow, C.D., 1996. Isolation and characterization of glutamine synthetase genes in Chlamydomonas reinhardtii. Plant Physiol. 112, 987-996.

Duclos-Vallee, J.C., Capel, F., Mabit, H., Petit, M.A., 1998. Phosphorylation of the hepatitis B virus core protein by glyceraldehyde-3phosphate dehydrogenase protein kinase activity. J Gen. Virol. 79, $1665-1670$.

Engel, M., Seifert, M., Theisinger, B., Seyfert, U., Welter, C., 1998. Glyceraldehyde-3-phosphate dehydrogenase and Nm23-H1/nucleoside diphosphate kinase A: two old enzymes combine for the novel Nm23 protein phosphotransferase function. J. Biol. Chem. 273, 20058-20065.

Georgopoulos, C., Welch, W.J., 1993. Role of the major heat shock proteins as molecular chaperones. Annu. Rev. Cell Biol. 9, 601-634.

Germ, M., Kreft, I., Osvald, J., 2005. Influence of UV-B exclusion and selenium treatment on photochemical efficiency of photosystem II, yield and respiratory potential in pumpkins (Cucurbita pepo L.). Plant Physiol. Biochem. 43, 445-448.

Ghisi, R., Trentin, A.R., Masi, A., Ferretti, M., 2002. Carbon and nitrogen metabolism in barley plants exposed to UV-B radiation. Physiol. Plant 116, 200-205.

Giavalisco, P., Nordhoff, E., Kreitler, T., Klöppel, K., Lehrach, H., Klose, J., Gobom, J., 2005. Proteome analysis of Arabidopsis thaliana by two-dimensional gel electrophoresis and matrix-assisted laser desorption/ionisation-time of flight mass spectrometry. Proteomics 5, 1902-1913.

Gleason, J.F., Bhartia, P.K., Herman, J.R., McPeters, R., Newman, P., Stolarski, R.S., Flynn, L., Labow, G., Larko, D., Seftor, C., Wellemeyer, C., Komhyr, W.D., Miller, A.J., Planet, W., 1993. Record low global ozone in 1992. Science 260, 523-526.

Green, R., Fluhr, R., 1995. UV-B-induced PR-1 accumulation is mediated by active oxygen species. Plant Cell 7, 203-212.

Izaguirre, M.M., Scopel, A.L., Baldwin, I.T., Ballare, C.L., 2003. Convergent responses to stress. Solar ultraviolet-B radiation and Manduca sexta herbivory elicit overlapping transcriptional responses in field-grown plants of Nicotiana longiflora. Plant Physiol. 132, 17551767.

Jordan, B.R., He, J., Chow, W.S., Anderson, J.M., 1992. Changes in mRNA levels and polypeptide subunits of ribulose 1,5-bisphosphate carboxylase in response to supplementary ultraviolet-B radiation. Plant Cell Environ. 15, 91-98.

Jordan, B.R., James, P.E., A-H-Macerness, S., 1998. Factors affecting UV-B-induced changes in Arabidopsis thaliana L. gene expression: the role of development, protective pigments and the chloroplast signal. Plant Cell Physiol. 39, 769-778.

Krizek, D.T., 2004. Influence of PAR and UV-A in determining plant sensitivity and photomorphogenic responses to UV-B radiation. Photochem. Photobiol. 79, 307-315.

Labra, M., Gianazza, E., Waitt, R., Eberini, I., Sozzi, A., Regondi, S., Grassi, F., Agradi, E., 2006. Zea mays L. protein changes in response to potassium dichromate treatments. Chemosphere 62, 1234-1244.

Leone, A., Piro, G., Leucci, M.R., Zacheo, G., Dalessandro, G., 2000. Membrane-cell wall-associated heat shock proteins in two genotypes of barley seedlings. Plant Biosyst. 134, 171-178.

Liakoura, V., Bornman, J.F., Karabourniotis, G., 2003. The ability of abaxial and adaxial epidermis of sun and shade leaves to attenuate UV-A and UV-B radiation in relation to the UV absorbing capacity of whole leaf methanolic extracts. Physiol. Plant 117, 33-43.

Middleton, E.H., Teramura, A.H., 1993. The role of flavonol glycosides and carotenoids in protecting soybean from ultraviolet-B damage. Plant Physiol. 103, 741-752.

Migge, A., Carrayol, E., Hirel, B., Lohmann, M., Meya, G., Becker, T.W., 1998. Two nitrite reductase isoforms are present in tomato cotyledons and are regulated differently by UV-A or UV-B light and during plant development. Planta 207, 229-234.

Molina, M.J., Rowland, F.S., 1974. Stratospheric sink for chloromethanes: chlorine atomic-catalysed destruction of ozone. Nature 249, $810-812$.
Nagy, E., Rigby, W.F.C., 1995. Glyceraldehyde-3-phosphate dehydrogenase selectively binds Au-rich RNA in the $\mathrm{NAD}^{+}$-binding region (Rossmann fold). J. Biol. Chem. 270, 2755-2769.

Natarajan, S., Xu, C., Caperna, T.J., Garrett, W.M., 2005. Comparison of protein solubilization methods suitable for proteomic analysis of soybean seed proteins. Anal. Biochem. 342, 214-220.

Newsholme, S.J., Maleeft, B.F., Steiner, S., Anderson, N.L., Schwartz, L.W., 2000. Two-dimensional electrophoresis of liver proteins: characterization of a drug-induced hepatomegaly in rats. Electrophoresis $21,2122-2128$.

Peng, Z.F., Strack, D., Baumert, A., Subramaniam, R., Goh, N.K., Chia, T.F., Tan, S.N., Chia, L.S., 2003. Antioxidant flavonoids from leaves of Polygonum hydropiper L.. Phytochemistry 62, 219-228.

Perkins, D.N., Pappin, D.J.C., Creasy, D.M., Cottrell, J.S., 1999. Probability-based protein identification by searching sequence databases using mass spectrometry data. Electrophoresis 20, 3551-3567.

Pinheiro, C., Kehr, J., Ricardo, C.P., 2005. Effects of water stress on lupin stem protein analysed by two-dimensional gel electrophoresis. Planta 221, 716-728.

Quaggiotti, S., Trentin, A.R., Vecchia, F.D., Ghisi, R., 2004. Response of maize (Zea mays L.) nitrate reductase to UV-B radiation. Plant Sci. 167, 107-116.

Rao, M.V., Palijyath, G., Ormrod, D.P., 1996. Ultraviolet-B- and ozoneinduced biochemical changes in antioxidant enzymes of Arabidopsis thaliana. Plant Physiol. 110, 125-136.

Reed, H.E., Teramura, A.H., Kenworthy, W.J., 1992. Ancestral US soybean cultivars characterized for tolerance to ultraviolet-B radiation. Crop Sci. 32, 1214-1219.

Robberecht, R., Caldwell, M.M., 1983. Protective mechanisms and acclimation to solar ultraviolet-B radiation in Oenothera stricta. Plant Cell Environ. 6, 477-485.

Ruhland, C.T., Xiong, F.S., Clark, W.D., Day, T.A., 2005. The influence of ultraviolet-B radiation on hydroxycinnamic acids, flavonoids and growth of Deschampsia antarctica during the springtime ozone depletion season in Antarctica. Photochem. Photobiol. 81, 1086-1093.

Russell, D.A., Wong, D.M.L., Sachs, M.M., 1990. The anaerobic response of soybean. Plant Physiol. 92, 401-407.

Sarnighausen, E., Wurtz, V., Heintz, D., Dorsselaer, A.V., Resk, R., 2004. Mapping of the Physcomitrella patens proteome. Phytochemistry 65, 1589-1607.

Schmelzer, E., Jahnen, W., Hahlbrock, K., 1988. In situ localisation of light-induced chalcone synthase mRNA, chalcone synthase, and flavonoid end products in epidermal cells of parsley leaves. PNAS 85, 2989-2993.

Searles, P.S., Flint, S.D., Caldwell, M.M., 2001. A meta-analysis of plant field studies stimulating stratospheric ozone depletion. Oecologia 127, $1-10$.

Shen, S., Jing, Y., Kuang, T., 2003. Proteomics approach to identify wound-response related proteins from rice leaf sheath. Proteomics 3, 527-535.

Sioud, M., Jespersen, L., 1996. Enhancement of hammerhead ri-bozyme catalysis by glyceraldehyde-3-phosphate dehydrogenase. J. Mol. Biol. 257, 775-789.

Sule, A., Vanrobaeys, F., Hajos, G., Beeumen, J.V., Devreese, B., 2004. Proteomic analysis of small heat shock protein isoforms in barley shoots. Phytochemistry 65, 1853-1863.

Sullivan, J.H., Teramura, A.H., 1990. Field study of the interaction between solar ultraviolet-B radiation and drought on photosynthesis and growth in soybean. Plant Physiol. 92, 141-146.

Surplus, S.L., Jordan, B.R., Murphy, A.M., Carr, J.P., Thomas, B., A-HMackerness, S., 1998. Ultraviolet-B-induced responses in Arabidopsis thaliana: role of salicylic acid and reactive oxygen species in the regulation of transcripts encoding photosynthetic and acidic pathogenesis-related proteins. Plant Cell Environ. 21, 685-694.

Ulm, R., Baumann, A., Oravecz, A., Mate, Z., Adam, E., Oakeley, E.J., Schafer, E., Nagy, F., 2004. Genome-wide analysis of gene expression reveals function of the bZIP transcription factor HY5 in the UV-B response of Arabidopsis. PNAS 101, 1397-1402. 
Wang, S.B., Chen, F., Sommerfeld, M., 2004. Proteomic analysis of molecular response to oxidative stress by the green alga Haematococcus pluvialis (Chlorophyceae). Planta 220, 17-29.

Watson, B.S., Asirvatham, V.S., Wang, L., Sumner, L.W., 2003. Mapping the proteome of barrel medic (Medicago truncatula). Plant Physiol. 131, 1104-1123.

Willekens, H., Camp, W.V., Montagu, M.V., Inze, D., Langebartels, C., Sandermann Jr., H., 1994. Ozone, sulfur dioxide, and ultraviolet B have similar effects on mRNA accumulation of antioxidant genes in Nicotiana plumbaginifolia L. Plant Physiol. 106, 1007-1014.

Wilson, K.A., McManus, M.T., Gordon, M.E., Jordan, T.W., 2002. The proteomics of senescence in leaves of white clover, Trifolium repens (L.). Proteomics 2, 1114-1122.

Xiong, F.S., Day, T.A., 2001. Effect of solar ultraviolet-B radiation during springtime ozone depletion on photosynthesis and biomass production of Antarctic vascular plants. Plant Physiol. 125, 738-751.
Xu, C., Garrett, W.M., Sullivan, J., Caperna, T.J., Natarajan, S., 2006. Separation and identification of soybean leaf proteins by twodimensional gel electrophoresis and mass spectrometry. Phytochemistry $67,2431-2440$.

Yan, S., Tang, Z., Su, W., Sun, W., 2005. Proteomic analysis of salt stressresponsive proteins in rice roots. Proteomics 5, 235-244.

Yan, S., Zhang, Q., Tang, Z., Su, W., Sun, W., 2006. Comparative proteomic analysis provides new insights into chilling stress responses in rice. Mol. Cell. Proteomics 5, 484- 496.

Yang, Y., Kwon, H.B., Peng, H.P., Shih, M.C., 1993. Stress responses and metabolic regulation of glyceraldehyde-3-phosphate dehydrogenase genes in Arabidopsis. Plant Physiol. 101, 209-216.

Yannarelli, G.G., Noriega, G.O., Batlle, A., Tomaro, M.L., 2006. Heme oxygenase up-regulation in ultraviolet-B irradiated soybean plants involves reactive oxygen species. Planta 224, 11541162 . 